\title{
Effects of knee extension with different speeds of movement on muscle and cerebral oxygenation
}

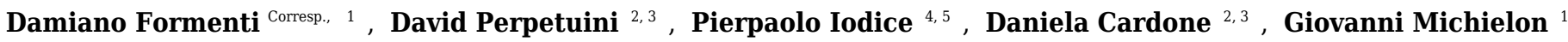 \\ , Raffaele Scurati ${ }^{1}$, Giampietro Alberti ${ }^{1}$, Arcangelo Merla ${ }^{2,3}$ \\ 1 Department of Biomedical Sciences for Health, Università degli Studi di Milano, Milan, Italy \\ 2 Department of Neuroscience, Imaging, and Clinical Sciences, University "G. d'Annunzio" Chieti-Pescara, Chieti, Italy \\ 3 Infrared Imaging Lab, Centro ITAB-Institute of Advanced Biomedical Technologies, University "G. d'Annunzio" Chieti-Pescara, Chieti, Italy \\ 4 Institute of Cognitive Sciences and Technologies, National Research Council, Rome, Italy \\ ${ }^{5}$ Centre d'Etude des Transformations des Activités Physiques et Sportives (CETAPS), University of Rouen Normandy, Mont-Saint-Aignan, France \\ Corresponding Author: Damiano Formenti \\ Email address: damiano.formenti@unimi.it
}

Background: One of the mechanisms responsible for enhancing muscular hypertrophy is the high metabolic stress associated with a reduced muscular oxygenation occurring during exercise, which can be achieved by reducing the speed of movement. Studies have tested that lowered muscle oxygenation artificially induced by an inflatable cuff, could provoke changes in prefrontal cortex oxygenation, hence, to central fatigue. It was hypothesized that 1 ) exercising with a slow speed of movement would result in greater increase in cerebral and greater decrease in muscle oxygenation compared with exercises of faster speed and 2) the amount of oxygenation increase in the ipsilateral prefrontal cortex would be lower than the contralateral one. Methods: An ISS Imagent frequency domain near infrared spectroscopy (NIRS) system was used to quantify oxygenation changes in the vastus lateralis muscle and prefrontal cortex (contra- and ipsilateral) during unilateral resistance exercises with different speeds of movement to voluntary fatigue. After 1 maximal repetition (1RM) test, eight subjects performed three sets of unilateral knee extensions ( $~ 50 \%$ of $1 \mathrm{RM})$, separated by 2 -min rest periods, following the pace of $1 \mathrm{~s}$, $3 s$ and $5 \mathrm{~s}$ for both concentric and eccentric phases, in a random order, during separate sessions. The amount of change for NIRS parameters for muscle $(\Delta \mathrm{Hb}$ : deoxyhemoglobin, $\triangle \mathrm{HbO}$ : oxyhemoglobin, $\Delta \mathrm{HbT}$ : total hemoglobin, $\Delta \mathrm{StO}_{2}$ : oxygen saturation) were quantified and compared between conditions and sets by two-way ANOVA RM. Differences in NIRS parameters between contra- and ipsilateral (lobe) prefrontal cortex and conditions were tested. Results: Exercising with slow speed of movement was associated to larger muscle deoxygenation than normal speed of movement, as revealed by significant interaction (set $x$ condition) for $\Delta \mathrm{Hb}(p=0.01)$, and by significant main effects of condition for $\Delta \mathrm{HbO}$ $(p=0.007)$ and $\Delta \mathrm{StO}_{2}(p=0.016)$. With regards to the prefrontal cortex, contralateral lobe 
showed larger oxygenation increase than the ipsilateral one for $\Delta \mathrm{Hb}, \Delta \mathrm{HbO}, \Delta \mathrm{HbT}, \Delta \mathrm{StO}_{2}$ in each set (main effect of lobe: $p<0.05$ ). Main effects of condition were significant only in set1 for all the parameters, and significant interaction lobe $x$ condition was found only for $\Delta \mathrm{Hb}$ in set1 $(p<0.05)$. Discussion: These findings provided evidence that speed of movement influences the amount of muscle oxygenation. Since the lack of oxygen in muscle is associated to increased metabolic stress, manipulating the speed of movement may be useful in planning resistance-training programs. Moreover, consistent oxygenation increases in both right and left prefrontal lobes were found, suggesting a complementary interaction between the ipsi- and contralateral prefrontal cortex, which also seems related to fatigue. 


\section{EFFECTS OF KNEE EXTENSION WITH DIFFERENT SPEEDS OF MOVEMENT ON MUSCLE AND CEREBRAL OXYGENATION}

Damiano Formenti ${ }^{1}$, David Perpetuini ${ }^{2}$, Pierpaolo Iodice ${ }^{3,4}$, Daniela Cardone $^{2}$, Giovanni Michielon $^{1}$, Raffaele Scurati ${ }^{1}$, Giampietro Alberti ${ }^{1}$, Arcangelo Merla ${ }^{2}$

${ }^{1}$ Department of Biomedical Sciences for Health, Università degli Studi di Milano, Milano, Italy.

${ }^{2}$ Infrared Imaging Lab, Centro ITAB-Institute of Advanced Biomedical Technologies and Department of Neuroscience, Imaging and Clinical Sciences, University "G. d'Annunzio" - ChietiPescara, Italy.

${ }^{3}$ Institute of Cognitive Sciences and Technologies, National Research Council, Via S. Martino della Battaglia 44, 00185 Rome, Italy.

${ }^{4}$ Centre d'Etude des Transformations des Activités Physiques et Sportives (CETAPS) EA 3832, University of Rouen Normandy, Mont-Saint-Aignan, France

Corresponding author:

Damiano Formenti, PhD

Department of Biomedical Sciences for Health

Università degli Studi di Milano

Via Kramer, 4/A

20129, Milano - Italy

Phone: +3902503 15158

Fax: +390250315152

E-mail: damiano.formenti@unimi.it 


\section{Abstract}

2 Background: One of the mechanisms responsible for enhancing muscular hypertrophy is the high

3 metabolic stress associated with a reduced muscular oxygenation occurring during exercise, which

4 can be achieved by reducing the speed of movement. Studies have tested that lowered muscle

5 oxygenation artificially induced by an inflatable cuff, could provoke changes in prefrontal cortex

6 oxygenation, hence, to central fatigue. It was hypothesized that 1) exercising with a slow speed of

7 movement would result in greater increase in cerebral and greater decrease in muscle oxygenation

8 compared with exercises of faster speed and 2) the amount of oxygenation increase in the

9 ipsilateral prefrontal cortex would be lower than the contralateral one.

10 Methods: An ISS Imagent frequency domain near infrared spectroscopy (NIRS) system was used

11 to quantify oxygenation changes in the vastus lateralis muscle and prefrontal cortex (contra- and

12 ipsilateral) during unilateral resistance exercises with different speeds of movement to voluntary

13 fatigue. After 1 maximal repetition (1RM) test, eight subjects performed three sets of unilateral

14 knee extensions ( $\sim 50 \%$ of $1 \mathrm{RM})$, separated by 2 -min rest periods, following the pace of $1 \mathrm{~s}, 3 \mathrm{~s}$ and

15 5s for both concentric and eccentric phases, in a random order, during separate sessions. The

16 amount of change for NIRS parameters for muscle $(\Delta \mathrm{Hb}$ : deoxyhemoglobin, $\Delta \mathrm{HbO}$ :

17 oxyhemoglobin, $\Delta \mathrm{HbT}$ : total hemoglobin, $\Delta \mathrm{StO}_{2}$ : oxygen saturation) were quantified and

18 compared between conditions and sets by two-way ANOVA RM. Differences in NIRS parameters

19 between contra- and ipsilateral (lobe) prefrontal cortex and conditions were tested.

20 Results: Exercising with slow speed of movement was associated to larger muscle deoxygenation

21 than normal speed of movement, as revealed by significant interaction (set x condition) for $\Delta \mathrm{Hb}$ 
$22(\mathrm{p}=0.01)$, and by significant main effects of condition for $\Delta \mathrm{HbO}(\mathrm{p}=0.007)$ and $\Delta \mathrm{StO}_{2}(\mathrm{p}=0.016)$.

23 With regards to the prefrontal cortex, contralateral lobe showed larger oxygenation increase than

24 the ipsilateral one for $\Delta \mathrm{Hb}, \Delta \mathrm{HbO}, \Delta \mathrm{HbT}, \Delta \mathrm{StO}_{2}$ in each set (main effect of lobe: $\mathrm{p}<0.05$ ). Main

25 effects of condition were significant only in set 1 for all the parameters, and significant interaction

26 lobe $\mathrm{x}$ condition was found only for $\Delta \mathrm{Hb}$ in set1 $(\mathrm{p}<0.05)$.

27 Discussion: These findings provided evidence that speed of movement influences the amount of 28 muscle oxygenation. Since the lack of oxygen in muscle is associated to increased metabolic stress,

29 manipulating the speed of movement may be useful in planning resistance-training programs.

30 Moreover, consistent oxygenation increases in both right and left prefrontal lobes were found,

31 suggesting a complementary interaction between the ipsi- and contralateral prefrontal cortex,

32 which also seems related to fatigue.

33

34

35

36

37

38 


\section{Introduction}

Maximizing hypertrophic response to resistance training can be reached by manipulation of exercise program variables, such as type and order of exercises, length of rest intervals, intensity

51 of maximal load, and training volume (Kraemer \& Ratamess, 2004). Recently, research attention has been devoted to repetition duration (i.e., the sum of the concentric, eccentric, and isometric actions of a repetition), a variable that has often been ignored (Headley et al., 2011). Substantially, current evidence suggests that hypertrophic outcomes are similar when training with repetition durations from 0.5 to $8.0 \mathrm{~s}$ to concentric failure (Schoenfeld, Ogborn \& Krieger, 2015). However, the review by Schoenfeld et al., (2015) considered studies employing protocols with different

57 intensities, which may have masked the role of repetition duration in muscle hypertrophy. Several studies have addressed the effects of low-intensity resistance exercise with slow movement (i.e., longer repetition duration) on muscular size and strength (Tanimoto \& Ishii, 2006; Tanimoto et al., 2008; Watanabe et al., 2013). This modality of resistance training is characterized by relatively low intensity ( $\sim 50 \%$ of 1 maximal repetition $(1 \mathrm{RM}))$ with repetition durations of $7 \mathrm{~s}(3 \mathrm{~s}$ eccentric,

$623 \mathrm{~s}$ concentric, and $1 \mathrm{~s}$ isometric contraction, no relaxation time). This has been shown to contribute to increased muscular hypertrophy and strength gains similar to conventional high intensity 64 resistance training with normal speed (1-2 s for eccentric and concentric phase), even with 65 relatively low intensity ( $\sim 50 \%$ of $1 \mathrm{RM})$ (Tanimoto \& Ishii, 2006; Tanimoto et al., 2008; Watanabe et al., 2013). In fact, one of the mechanisms responsible for enhancing muscular hypertrophy is

67 the lowered peripheral muscular oxygenation occurring during exercise (Tamaki et al., 1994), 
68 which can be obtained naturally by reducing the speed of movement (Tanimoto, Madarame \&

69 Ishii, 2005; Tanimoto \& Ishii, 2006). It has been proposed that muscle hypertrophy is likely

70 attributed to mechanical tension and to the increased levels of metabolic stress within muscle (as

71 a result of the intramuscular environment with reduced oxygenation) (Pearson \& Hussain, 2015),

72 which in turn stimulates growth hormone $(\mathrm{GH})$ secretion (Takarada et al., 2000), cell swelling

73 (Loenneke et al., 2012), production of reaction oxygen species (ROS) (Kawada \& Ishii, 2005), and

74 increased recruitment of fast-twitch muscle fibers (Moritani et al., 1992). A much lower peripheral

75 muscle oxygenation can be likely achieved by further lowering the speed of movement, thus

76 increasing the repetition duration and maximizing the hypertrophic response. Furthermore, it has

77 been suggested that a reduced muscular oxygenation elicits early recruitment of fast twitch-fibers

78 because of early fatigue (Husmann et al., 2017). Moreover, a reduced muscular oxygenation during

79 resistance exercise to fatigue has been found to be related with increase in deoxyhemoglobin

80 concentration and lower increase in oxyhemoglobin concentration in prefrontal cortex, thus

81 suggesting that the ability to sustain exercise to exhaustion is related to both muscle and cerebral

82 oxygenation (Ganesan et al., 2015). In fact, a decrease in oxygenation of the prefrontal cortex at

83 exhaustion may lead to the decision to stop exercising (Shibuya \& Tachi, 2006; Subudhi, Dimmen

84 \& Roach, 2007; Subudhi et al., 2009).

85 Recent studies have examined the cerebral and muscle oxygenation changes during sustained 86 isometric (Pereira, Gomes \& Bhambhani, 2009) and dynamic knee extension to voluntary fatigue

87 (Matsuura et al., 2011). It was found that fatigue during unilateral knee extensions performed under

88 isometric and isotonic conditions was not related to a decline in neuronal activation but rather

89 mediated peripherally in the exercising muscle (Pereira, Gomes \& Bhambhani, 2009). In fact,

90 cerebral oxygenation increased systematically during the exercise with no signs of attenuation. 
91 Hence, muscle deoxygenation may be directly responsible for the inability to sustain adequate

92 muscle contractions at exhaustion and to inhibit central motor output via peripheral feedback

93 system (Gandevia, 2001). On the other hand, since cerebral oxygenation reflects modulations in

94 cerebral functional activation (Colier et al., 1999), a decreased cerebral oxygenation may affect

95 maximal exercise capacity, which highlights the key role of cerebral oxygenation in modulating

96 motor output at exhaustion (Shibuya \& Tachi, 2006; Subudhi, Dimmen \& Roach, 2007; Subudhi

97 et al., 2009).

98 Furthermore, it has been demonstrated that during handgrip exercise the ipsilateral and

99 contralateral frontal lobes have different oxygenation dynamics (Kuboyama \& Shibuya, 2015) and

100 blood flow (Fernandes et al., 2016). It is licit to suppose that unilateral resistance training could

101 induce differences in contralateral and ipsilateral frontal cortical oxygenation.

102 Hitherto, to the best of our knowledge, there are no studies that have investigated whether

103 resistance exercise with different repetition durations (and different speed of movement) would

104 induce differences in muscle oxygenation, as well as differences in ipsilateral and contralateral

105 prefrontal cortex oxygenation. We hypothesized that measurements of muscle hemodynamic and

106 oxygenation during resistance exercises with different repetition durations with the same intensity

107 would provide new insights into these processes. In this study we monitored muscle and prefrontal

108 cortex oxygenation through Near Infrared Spectroscopy (NIRS) to investigate tissue oxygenation

109 responses to resistance exercise to voluntary fatigue, performed with different speeds of

110 movement.

111 Therefore, the purposes of the current study were: 1) to investigate the effect of resistance exercises

112 with different speeds of movement (i.e., different repetition duration) on muscle and cerebral 
113 oxygenation; 2) to investigate the difference in oxygenation change between the ipsilateral and

114 contralateral prefrontal cortex during resistance exercises to voluntary fatigue.

115 We hypothesized that: 1) the exercise with slow speed of movement (longer repetition duration)

116 would result in greater increase in cerebral oxygenation and greater decrease in muscle

117 oxygenation compared with exercises of faster speed; 2) during the exercises, the amount of

118 oxygenation increase in the ipsilateral prefrontal cortex would be lower compared to the

119 contralateral one.

120

121 Material and methods

122 Participants

123 Eight young healthy female subjects, who were not involved in regular resistance training

124 programs at the time of the study, volunteered to participate. The subjects were sub-elite futsal

125 players. The choice of a futsal team permitted to have high interindividual homogeneity of

126 anthropometric characteristics and training conditions. Their age, body mass and stature were

$12724.8 \pm 4.9$ years, $63.3 \pm 7.2 \mathrm{~kg}$ and $164 \pm 4.7 \mathrm{~cm}($ mean $\pm \mathrm{SD})$. None of the participants reported recent

128 lower limb injuries. According to the declaration of Helsinki, the study was approved by the

129 Ethical Committee of the Università degli Studi di Milano (Approval number: 2/12). After a

130 thorough explanation of the protocol, the subjects provided informed written consent to participate

131 in this study.

132 The sample size was calculated based on a power analysis [G*Power 3.1.9.2

133 (www.gpower.hlu.de/en.html)] conducted on the effect size (Cohen's f, values varying between

1340.85 and 0.89 ) calculated by data reported in a previous study on the same topic (Ganesan et al.,

135 2015). The analysis was based on the following parameters: $\eta_{\mathrm{p}}{ }^{2}=0.43$; alpha $=0.05$; power $=0.95$; 
136 number of groups / measures: depends on the specific analysis (Cohen, 1988; Richardson, 2011).

137 The results of these analyses indicated a required sample size ranging from 7 to 9 participants; we

138 observed 8 to be a critical number of participants, as the effect size and observed power remains

139 stable above this number.

\section{Experimental protocol}

142 We used a within-subjects design, so that each subject served as their own control. The experiment 143 comprised four different sessions, each one separated by five days. After a preliminary session,

144 the three experimental sessions, each one involving different conditions - denoted as 1 s exercise,

$1453 \mathrm{~s}$ exercise and $5 \mathrm{~s}$ exercise - were randomized. The subjects were instructed to refrain from

146 strenuous physical activity in the two days before the trials and abstained from consuming

147 alcoholic or caffeine-containing products for a 4-h period before the start of the experiment. All

148 sessions were scheduled in the late morning to mitigate possible effects due to circadian rhythm

149 variations. A schematic representation of the experimental design is shown in Figure 1.

***Insert Figure 1 near here***

Preliminary session

154 The purpose of the preliminary session was to collect anthropometric measurements, to find out

155 the load of maximal repetition (1RM) in knee extension of the dominant leg, as well as to

156 familiarize the subjects with different speeds of movement (i.e., $1 \mathrm{~s}, 3 \mathrm{~s}$ and 5 s exercises). All the

157 subjects were right-leg dominant. Participants warmed up by completing a number of submaximal

158 repetitions $(\sim 15)$ at approximately $20 \%$ of the perceived 1 RM. After $60 \mathrm{~s}$ of rest, an initial weight 
159 within the subject's perceived capacity ( $\sim 50 \%-70 \%)$ was chosen. Resistance was progressively

160 increased by $10.0 \%-20.0 \%$ from the previous successful attempt until the subjects could not

161 complete the single repetition throughout the full range of motion. The range of motion was set to

$162105^{\circ}$ (i.e., from starting position of $75^{\circ}$ to ending position of $180^{\circ}$ ). The $1 \mathrm{RM}$ was determined as

163 the maximum weight that the subjects were able to lift once. A rest period of $300 \mathrm{~s}$ was given to

164 participants between successive trials, and in all of the cases the $1 \mathrm{RM}$ was obtained within five

165 trials. After this assessment, the subjects were familiarized with the resistance exercise protocol

166 used in the subsequent three testing sessions. Participants performed three sets of knee extensions,

167 using $\sim 30 \%$ of their 1 RM, with each set lasting approximately $60 \mathrm{~s}$. Each set was performed using

168 a specific speed of movement $(1 \mathrm{~s} / 1 \mathrm{~s}, 3 \mathrm{~s} / 3 \mathrm{~s}, 5 \mathrm{~s} / 5 \mathrm{~s}$ for concentric and eccentric condition

169 respectively, randomly administrated) with the aid of a metronome.

170

\section{Testing visits: 1 s, 3s and 5s exercise sessions}

172 Before the trials, subjects performed a standardized warm-up, i.e., 10 min of walking on a

173 treadmill. After that, and before the beginning of the exercises, subjects acclimated to the room

174 conditions (temperature $22-24^{\circ} \mathrm{C}$; relative humidity $50 \pm 5 \%$; no direct ventilation and constant

175 intensity of light) for $10 \mathrm{~min}$, at rest, remaining seated on the knee extension machine (Teca srl,

176 Ortona, Italy). Then, subjects underwent either the $1 \mathrm{~s}, 3 \mathrm{~s}$ or $5 \mathrm{~s}$ knee extension exercises (the three

177 conditions were randomized and separated by at least 5 days) using an intensity of $\sim 50 \%$ of 1 RM.

178 Each exercise session consisted of three sets of knee extensions with an inter-set rest period of 3

$179 \min$.

180 In either session, subjects repeated the movement until exhaustion (concentric failure) following

181 the $1 \mathrm{~s} / 3 \mathrm{~s} / 5 \mathrm{~s}$ pace for each phase of contraction. A metronome was used to ensure that each 
182 repetition duration lasted $2 \mathrm{~s}, 6 \mathrm{~s}$, and $10 \mathrm{~s}$ for each of the $1 \mathrm{~s} / 3 \mathrm{~s} / 5 \mathrm{~s}$ conditions respectively. Shoulder

183 straps were used to stabilize the subjects and to minimize the use of trunk muscles during the

184 exercises. At the end of each exercise set, subjects were asked to provide a rating of perceived

185 exertion (RPE), expressed as a number between 1 and 10 (Day et al., 2004).

186

187 Near Infrared Spectroscopy Measurements

188 Muscle and cerebral oxygenation were assessed using a frequency-domain near-infrared

189 spectroscopy machine, with two wavelengths of near-infrared light (690 and $830 \mathrm{~nm})$, at a

190 sampling rate of $5 \mathrm{~Hz}$ (ISS Imagent, ISS, Champaign, IL). A multi-distance optical probe,

191 configured with one optical detector and four optical source fibers, was attached to the muscle.

192 The fibers were positioned on a custom-made probe such that there are four source-detector

193 separation distances $(2.0,2.5,3.0,3.5 \mathrm{~cm})$ for each wavelength. The four sources were on the same

194 line of the detector. The probe was positioned on the belly of the vastus lateralis of the right limb, 195 approximately $12 \mathrm{~cm}$ from the lateral epicondyle of the femur, as used in previous resistance

196 exercise studies (Gomes, Matsuura \& Bhambhani, 2012; Yeung et al., 2016). To ensure the

197 reproduction of the probe position in the following procedures, pen-marks were made around the

198 margins of the probe. The probe was secured with tape and black bandages were wrapped around

199 the leg to block background light. Skinfold thickness at the site of probe placement (on vastus

200 lateralis) was measured by a skinfold caliper, in order to determine adipose tissue thickness,

201 defined as skinfold thickness/2. The source-detector separation distances of the probes allowed to

202 investigate up to $18 \mathrm{~mm}$ of depth. The adipose tissue thickness $(7.2 \pm 1.2 \mathrm{~mm})$ was lower than the

203 NIRS light penetration depth, and therefore NIRS measurements were not influenced by adipose

204 tissue (van Beekvelt et al., 2001). 
205 In order to record the oxygenation of both the contralateral and ipsilateral prefrontal cortex 206 (Chiarelli et al., 2016), a probe composed of two detectors and eight sources with the same source-

207 detector distance configuration of the muscle probe was used. The center of the probe was 208 positioned in correspondence with the Fp point (fronto polar), which is a marker defined in the 1020920 international system for the placement of the Electroencephalography (EEG) probes. It is placed 210 proximally to the nasion (root of nose), in a position equating to $10 \%$ of the overall distance 211 between the nasion and the inion (external occipital protuberance) (Homan, Herman \& Purdy, 212 1987; Chiarelli et al., 2015b).

213 The two detectors were $3 \mathrm{~cm}$ distant from the Fp point, one on the left and one on the right side of

214 the prefrontal cortex. Participants were instructed to keep their head as still as possible during 215 exercise, in order to minimize the possible motion artefacts of the cerebral NIRS signal.

216 Before the initiation of the data recording, the near infrared spectroscopy system was calibrated 217 with a factory-manufactured calibration optical phantom with known optical properties 218 (absorption and scattering coefficients) according to the procedure proposed by Hueber and 219 colleagues (Hueber et al., 1999), which was implemented in the software of the ISS Imagent 220 system. Absolute values of oxyhemoglobin, $(\mathrm{HbO})$ deoxyhemoglobin $(\mathrm{Hb})$ and total hemoglobin 221 (HbT) concentration in the muscle and in the prefrontal cortex were monitored continuously 222 through the testing procedure. The $\mathrm{StO}_{2}$ was calculated as $(\mathrm{HbO}) /(\mathrm{HbT})$ and was expressed as 223 percentage.

$224 \mathrm{HbO}, \mathrm{Hb}, \mathrm{HbT}$ and $\mathrm{StO}_{2}$ signals were low-pass filtered (3 ${ }^{\text {rd }}$ order Butterworth filter, cutoff 225 frequency: 0.14 Hz) (Bandrivskyy et al., 2004; Kvandal et al., 2006) in order to eliminate some 226 systemic noises. Then, we used a Savitzky-Golay Filter ( $4^{\text {th }}$ order, frame size: 121) (Ferrante et al., 
227 2009) to smooth the signals, thus eliminating the fluctuations of the signals due to the movement

228 (Chiarelli et al., 2015a).

229 To assess exercise-associated oxygenation changes in the muscle, as well as changes in the left

230 and right prefrontal cortices, we calculated $\Delta \mathrm{HbO}, \Delta \mathrm{Hb}, \Delta \mathrm{HbT}$ and $\Delta \mathrm{StO}_{2}$ during execution of the

231 exercise. These parameters were calculated as the difference between the absolute maximum

232 variations in hemoglobin from the baseline. Absolute maximum variations were calculated as the

233 mean values of $5 \mathrm{~s}$ around the peak value $( \pm 2.5 \mathrm{~s})$, whereas the baseline was calculated as the

234 mean value from the last $5 \mathrm{~s}$ to $1 \mathrm{~s}$ before the onset of each exercise set.

235

236 Statistical analysis

237 Data were expressed as mean \pm SD. The normality of the distribution of the data was checked by 238 the Shapiro-Wilk's normality test. All the data met the assumption of normality.

239 A two-way (i.e., set and condition) analysis of variance (ANOVA) with repeated measures on two

240 factors was used to investigate the effect of conditions throughout sets in the number of repetitions

241 and in RPE.

242 To test whether the amount of muscle oxygenation change in set1, set2 and set3 was influenced

243 by the speed of movement, we used two-way (i.e., set and condition) ANOVA with repeated

244 measures on two factors. To test whether the amount of cerebral oxygenation change in the 245 contralateral and ipsilateral prefrontal lobe was influenced by the speed of movement, we used a 246 two-way (i.e., lobe and condition) ANOVA with repeated measures on two factors for each set.

247 As a measure of effect size for ANOVA, partial eta squared $\left(\eta_{\mathrm{P}}^{2}\right)$ was reported. 
248 Least significant difference (LSD) post-hoc analyses were used to compare pairs of means.

249 Statistical analysis was performed using Graphpad Prism software (version 7.0, Graphpad, San

250 Diego, CA). A p-value lower than 0.05 was considered statistically significant.

251

252 Results

253 Exercise parameters

254 The effect of condition ( $1 \mathrm{~s}, 3 \mathrm{~s}$ and $5 \mathrm{~s}$ exercise) on number of repetitions throughout the three sets

255 is represented in Figure 2A, together with pairwise comparisons between conditions provided by 256 LSD.

257 The two-way ANOVA repeated measures revealed a significant interaction (set $\mathrm{x}$ condition) for 258 the number of repetitions $\left(\mathrm{F}_{4,28}=3.74, \mathrm{p}=0.014, \eta_{\mathrm{P}}{ }^{2}=0.29\right)$. This implies that exercising with 259 different speed of execution (condition) until fatigue induces different number of repetitions across 260 the sets. Furthermore, the main effect of set $\left(\mathrm{F}_{2,14}=13.53, \mathrm{p}<0.001, \eta_{\mathrm{P}}{ }^{2}=0.44\right)$ and the main effect 261 of condition were significant $\left(\mathrm{F}_{2,14}=85.53, \mathrm{p}<0.001, \eta_{\mathrm{p}}{ }^{2}=0.71\right)$.

262 No significant interaction (set $x$ condition) was found for $\operatorname{RPE}\left(\mathrm{F}_{4,28}=1.615, \mathrm{p}=0.198, \eta_{\mathrm{P}}^{2}=0.09\right)$, 263 whereas the main effect of set $\left(\mathrm{F}_{2,14}=45.71, \mathrm{p}<0.0001, \eta_{\mathrm{p}}{ }^{2}=0.63\right)$ and condition $\left(\mathrm{F}_{2,14}=8.48\right.$, $264 \mathrm{p}=0.004, \eta_{\mathrm{P}}^{2}=0.40$ ) were found to be significant. In general, RPE was higher in the $5 \mathrm{~s}$ exercise, 265 with respect to $3 \mathrm{~s}$ and $1 \mathrm{~s}$ exercise, increasing from set1 to set3. The effect of condition $(1 \mathrm{~s}, 3 \mathrm{~s}$ and 2665 s exercise) on RPE across the three sets is represented in Figure 2B, together with pairwise 267 comparisons between conditions provided by LSD. 268 


\section{Muscle oxygenation}

272 The acute effects of knee extension exercise on muscle hemodynamic responses ( $\mathrm{Hb}$ and $\mathrm{HbO})$ in

273 a representative subject is illustrated in Figure 3A. Overall, the time courses were similar in all the

274 three exercise conditions ( $1 \mathrm{~s}, 3 \mathrm{~s}$ and 5 s exercise) for that particular variable, and are therefore

275 described only in general terms. At the beginning of the exercise (in each set), Hb increased very

276 rapidly and tended to level off or decrease slightly towards the end of each set. At the cessation of

277 the exercise, during recovery, $\mathrm{Hb}$ began to decrease rapidly until reaching the baseline value. In

278 parallel with $\mathrm{Hb}, \mathrm{HbO}$ decreased rapidly from the initiation of the exercise, levelling off at the end

279 of each exercise set and began to increase in the recovery returning to the baseline.

280

281

***Insert Figure 3 near here***

282

283 The amount of $\mathrm{Hb}$ and $\mathrm{HbO}$ change, which was quantified by $\Delta \mathrm{Hb}$ and $\Delta \mathrm{HbO}$, were different 284 between conditions across sets. In fact, the two-way ANOVA repeated measures revealed a 285 significant interaction (set $\mathrm{x}$ condition) for $\Delta \mathrm{Hb}\left(\mathrm{F}_{4,28}=4.036, \mathrm{p}=0.01, \eta_{\mathrm{P}}{ }^{2}=0.30\right)$, implying that 286 exercising with different speed of movement (condition) until fatigue induces different amount of 287 deoxygenation. The main effect of set was significant $\left(\mathrm{F}_{2,14}=10.14, \mathrm{p}=0.001, \eta_{\mathrm{P}}{ }^{2}=0.44\right)$, whereas 288 the main effect of condition was not significant $\left(\mathrm{F}_{2,14}=3.18, \mathrm{p}=0.07, \eta_{\mathrm{P}}{ }^{2}=0.13\right)$ (Figure $\left.4 \mathrm{~A}\right)$.

289 No significant interaction (set $\mathrm{x}$ condition) was found for $\Delta \mathrm{HbO}\left(\mathrm{F}_{4,28}=0.667, \mathrm{p}=0.619, \eta_{\mathrm{P}}^{2}=0.03\right)$, 290 but there was a significant main effect of set $\left(F_{2,14}=7.404, p=0.006, \eta_{\mathrm{P}}{ }^{2}=0.39\right)$ and condition $291\left(\mathrm{~F}_{2,14}=6.995, \mathrm{p}=0.007, \eta_{\mathrm{P}}{ }^{2}=0.38\right)$. Specifically, $\Delta \mathrm{HbO}$ was higher in $1 \mathrm{~s}$ set1 than $1 \mathrm{~s}$ set3 $(\mathrm{p}=0.01)$, 292 and it was higher in $5 \mathrm{~s} \operatorname{set} 1>5 \mathrm{~s}$ set3 $(\mathrm{p}=0.02)$. Concerning the main effect of condition, $\Delta \mathrm{HbO}$ in 293 1s was lower than $5 \mathrm{~s}$ in set1 ( $\mathrm{p}=0.006)$, and than $3 \mathrm{~s}$ and $5 \mathrm{~s}$ in set2 and set $3(\mathrm{p}<0.05)$ (Figure 4B). 
294 For $\Delta \mathrm{HbT}$, no significant interaction (set $\mathrm{x}$ condition) was found for $\Delta \mathrm{HbT}\left(\mathrm{F}_{4,28}=2.09, \mathrm{p}=0.108\right.$, $\left.295 \eta_{\mathrm{P}}{ }^{2}=0.08\right)$, as well as no significant main effect of set $\left(\mathrm{F}_{2,14}=0.253, \mathrm{p}=0.779, \eta_{\mathrm{P}}{ }^{2}=0.006\right)$ and 296 condition $\left(\mathrm{F}_{2,14}=0.109, \mathrm{p}=0.897, \eta_{\mathrm{P}}{ }^{2}=0.001\right)$ (Figure 4C).

297 No significant interaction (set $\mathrm{x}$ condition) was found for $\Delta \mathrm{StO}_{2}\left(\mathrm{~F}_{4,28}=0.281, \mathrm{p}=0.887, \eta_{\mathrm{P}}{ }^{2}=0.001\right.$ ), 298 whereas the main effect of $\operatorname{set}\left(\mathrm{F}_{2,14}=12.58, \mathrm{p}<0.001, \eta_{\mathrm{p}}{ }^{2}=0.45\right)$ and condition $\left(\mathrm{F}_{2,14}=5.63, \mathrm{p}=0.016\right.$, $299 \eta_{\mathrm{P}}{ }^{2}=0.31$ ) were found to be significant. Specifically, $\Delta \mathrm{StO}_{2}$ was higher in $1 \mathrm{~s}$ set1 than $1 \mathrm{~s}$ set2 $300(\mathrm{p}=0.003)$ and $1 \mathrm{~s}$ set $3(\mathrm{p}=0.002) ; 3 \mathrm{~s}$ set 1 was higher than $3 \mathrm{~s}$ set $3(\mathrm{p}=0.013) ; 5 \mathrm{~s}$ set 1 was higher 301 than $5 \mathrm{~s}$ set2 $(\mathrm{p}=0.007)$ and $5 \mathrm{~s} \operatorname{set} 3(\mathrm{p}=0.003)$. With regards to the main effect of condition, $\Delta \mathrm{StO}_{2}$ 302 was lower in $1 \mathrm{~s}$ than $3 \mathrm{~s}$ and $5 \mathrm{~s}$ in set1, set2 and set3 $(\mathrm{p}<0.05)$ (Figure 4D).

***Insert Figure 4 near here ${ }^{* * *}$

\section{Cerebral oxygenation}

307 The acute effects of knee extension exercise on contralateral and ipsilateral prefrontal cortex

308 hemodynamic responses $(\mathrm{Hb}$ and $\mathrm{HbO})$ in a representative subject is illustrated in Figure 3B.

309 Overall, as in muscle, the time courses were similar in all three exercise conditions ( $1 \mathrm{~s}, 3 \mathrm{~s}$ and $5 \mathrm{~s}$

310 exercise), in both the left and right prefrontal cortex, and in each set, for that particular variable,

311 and are therefore described only in general terms. At the beginning of the exercise (in each set),

$312 \mathrm{Hb}$ decreased slightly and levelled off towards the end of each set, whereas $\mathrm{HbO}$ increased during 313 each exercise set.

314 A two-way ANOVA repeated measures (lobe $\mathrm{x}$ condition) was performed in each of the three sets

315 to investigate the effects of lobe and condition for the oxygenation parameters $(\Delta \mathrm{Hb}, \Delta \mathrm{HbO}$,

$316 \Delta \mathrm{HbT}, \Delta \mathrm{StO}_{2}$ ), and their results are reported in Table 1. Mean values $\pm \mathrm{SD}$ of $\Delta \mathrm{Hb}, \Delta \mathrm{HbO}, \Delta \mathrm{HbT}$, 
$317 \Delta \mathrm{StO}_{2}$ in each condition and in each set in the two prefrontal lobes are illustrated in Figure 5,

318 together with pairwise comparisons.

319

\section{Discussion}

324 The main findings of this study were as follows: 1) exercising with slow speed of movement

325 induces higher muscle deoxygenation than exercising with normal speed of movement; 2)

326 exercise-associated oxygenation changes were higher in the contralateral compared to the

327 ipsilateral prefrontal cortex.

\section{Muscle oxygenation}

330 Consistent with the study hypothesis, exercising with different speeds of movement (1s, $3 \mathrm{~s}$ and $5 \mathrm{~s}$

331 exercise: condition) until fatigue induced different amount of change in muscle oxygenation. In

332 fact, differences between conditions were found in each of the three sets for $\Delta \mathrm{HbO}$ and $\Delta \mathrm{StO}_{2} . \mathrm{A}$

333 significant (condition $\mathrm{x}$ set) interaction was also found for $\Delta \mathrm{Hb}$.

334 In the present study we found that exercising with slow speed of movement induced larger muscle 335 deoxygenation compared to exercising with normal speed of movement (i.e., 1s exercise). Many

336 lines of evidence point to such a conclusion. The first observation is that the amount of change in

337 oxygenation was larger in 5 s than $3 \mathrm{~s}$ exercise and in 3 s than 1 s exercise (Figure 3 ). Accordingly,

338 the results of the two-way ANOVA for repeated measures revealed a significant interaction 
339 between condition and set for $\Delta \mathrm{Hb}$ and a main effect of condition for $\Delta \mathrm{HbO}$, thus indicating that

340 speed of movement has an effect on the amount of oxygenation change (Figure 4).

341 Our results are in line with the study by Tanimoto and Ishii (Tanimoto \& Ishii, 2006), which

342 investigated the acute oxygenation changes in the vastus lateralis during a knee extension exercise

343 with different speeds of execution (different repetition durations). It was found that exercising with

344 slow speed of movement ( $3 \mathrm{~s}$ for concentric and eccentric phase) with relatively low intensity $(\sim 50$

$345 \%$ of 1RM) elicited significantly larger muscle deoxygenation than the other two protocols, which

346 were performed with normal speed of movement ( $1 \mathrm{~s}$ for each phase) and different intensities $(\sim 50$

$347 \%$ of $1 \mathrm{RM}$, and $\sim 80 \%$ of $1 \mathrm{RM})$. In that study, a 12-week period of resistance exercise with slow

348 speed of movement was also accompanied by positive effects on muscular size and strength, which

349 were similar to those obtained by exercise with normal speed. Based on these findings, the authors

350 suggested that muscle hypertrophy may be mediated by the hypoxic condition of the intramuscular

351 environment, increasing metabolic stress and stimulating systemic hormone secretion, cell

352 swelling, production of ROS and increased recruitment of fast-twitch fibers (Pearson \& Hussain, 353 2015).

354 In addition, to investigate muscle oxygenation change in exercise with a slow speed of movement

355 (3s for each phase) compared to an exercise with a normal speed movement (1s for each phase) -

356 already addressed by (Tanimoto \& Ishii, 2006) - we also investigated oxygenation change

357 associated to an exercise with an even slower speed of movement (i.e., 5s for each phase). We

358 found that the 5 s exercise was associated with larger muscle deoxygenation compared to the $1 \mathrm{~s}$

359 exercise, as shown by the significant interaction for $\Delta \mathrm{Hb}$, and the significant main effect of

360 condition for $\Delta \mathrm{HbO}$, and $\Delta \mathrm{StO}_{2}$ (Figure 4). Specifically, significant differences between

361 conditions were only found between $1 \mathrm{~s}$ and $3 \mathrm{~s}$ exercise (for set 2 and set 3 ), and between $1 \mathrm{~s}$ and $5 \mathrm{~s}$ 
362 exercise (for each set). No significant differences were found between $3 \mathrm{~s}$ and $5 \mathrm{~s}$ exercise for all of

363 the parameters, which seems to indicate that exercising with an even slower speed of movement

364 than $3 \mathrm{~s}$ for each phase did not elicit an even lower muscle oxygenation (Figure 4). Moreover, $5 \mathrm{~s}$

365 exercise was associated with a lower number of repetitions and higher RPE in each set, with respect

366 to both $1 \mathrm{~s}$ and $3 \mathrm{~s}$ exercise (Figure 2).

367 It is important to notice that no differences between conditions were found for $\Delta \mathrm{HbT}$, which can

368 be considered an indirect measure of change in blood flow (Felici et al., 2009). Given that $\Delta \mathrm{HbT}$

369 was similar among conditions, we speculate that the blood flow modifications induced by the

370 exercise were similar among conditions. Therefore, exercising with slow movement caused greater

371 deoxygenation in muscles, which was probably due to larger oxygen consumption, while the

372 changes in blood flow remained the same as during exercise with normal speed of movement.

373 The significant main effect of set found for $\Delta \mathrm{HbO}$ and $\Delta \mathrm{StO}_{2}$ demonstrated a significant decrement

374 of oxygenation change from set 1 to set3. This can be explained by exercise-related fatigue

375 throughout the whole exercise session. The decrement of changes in oxygenation from set 1 to set3

376 was also consistent with the exercise parameters (number of repetitions and RPE) which changed

377 across the training session. In particular, the number of repetitions were higher in set1, decreasing

378 in set2 and in set3, and accordingly, RPE increased from set 1 to set3. These findings are consistent

379 with the data presented in a recent study (Ganesan et al., 2015). In that study, the authors

380 investigated the oxygenation changes in the vastus medialis muscle and the prefrontal cortex of

381 the brain during three sets of knee extension exercise, with and without blood flow restriction

382 induced by an inflatable cuff. Although no between sets comparisons were shown, the number of

383 repetitions decreased from set1 to set3, and accordingly RPE increased from set1 to set3. 
384 Our results demonstrated that exercising with a slow speed of movement induced higher amount

385 of muscle deoxygenation than normal speed of movement (1s for each phase, i.e., $2 \mathrm{~s}$ for each

386 repetition). It is worth noticing that, despite the discrepancy between the two experimental

387 conditions (i.e., slow speed of movement in our study vs. blood flow restriction with an inflatable

388 cuff in the study by Ganesan and colleagues), these two protocols elicited a significant decrease in

389 muscle oxygenation (Ganesan et al., 2015). It has been suggested that mechanical tension and

390 metabolic stress are the two primary mechanisms for stimulating muscle hypertrophy, the latter

391 magnified under conditions of lack of oxygen within an intramuscular environment (Pearson \&

392 Hussain, 2015). Metabolic stress mediates muscle growth via a series of secondary mechanisms,

393 such as increased hormone productions (Takarada et al., 2000), increased fast-twitch fibers

394 recruitment (Moritani et al., 1992), cell swelling (Loenneke et al., 2012) and increased ROS

395 production (Kawada \& Ishii, 2005). Although resistance training with slow speed of movement is

396 characterized by low intensity, as that employed in the present study, the higher deoxygenation

397 reached by such condition may be considered an additional stimulus for inducing high metabolic

398 stress, thus maximizing one of the two primary mechanisms by which hypertrophy is stimulated.

399 Therefore, the manipulation of speed of movement, and the related repetition duration, should be

400 considered when planning resistance training to maximize metabolic stress within intramuscular

401 environment.

402

403 Cerebral oxygenation

404 We found that exercising with slow speed of movement (5s exercise and 3 s exercise) induced

405 larger changes in both the left and right prefrontal cortex oxygenation, compared to exercising

406 with normal speed of movement (1s exercise). Such changes were more pronounced in the 
407 contralateral prefrontal lobe. The fact that exercising with slow speed of movement elicited greater 408 oxygenation change in both the contralateral and ipsilateral prefrontal cortices, confirmed that

409 fatigue seems to be mediated not only peripherally, but also centrally (Ganesan et al., 2015). This

410 is particularly evident for the set1, in which a significant interaction (lobe $\mathrm{x}$ condition) was found

411 for $\Delta \mathrm{Hb}$. Moreover, in the contralateral prefrontal cortex, the 1s exercise resulted in lower

412 oxygenation change than $3 \mathrm{~s}$ exercise and $5 \mathrm{~s}$ exercise $\left(\Delta \mathrm{HbO}, \Delta \mathrm{StO}_{2}, \Delta \mathrm{HbT}\right)$. It is worth pointing

413 out that, despite the significant main effect of lobe, LSD pairwise comparisons revealed significant

414 differences between lobes only in the $3 \mathrm{~s}$ and 5 s exercise conditions $\left(\Delta \mathrm{HbO}, \Delta \mathrm{StO}_{2}, \Delta \mathrm{HbT}\right)$ (Figure

415 5). Conversely, in set2 and set3, no significant main effects of conditions were found, whereas

416 there were significant main effects of lobe (Table 1). The fact that in set2 and in set3 no differences

417 between conditions were found for all the parameters may be explained by physiological

418 mechanisms related to change in blood flow, which are still very poorly investigated. In fact,

419 studies pertaining to the effect of skin blood flow on cerebral oxygenation during exercise focused

420 only on a walking treadmill task (Kohno et al., 2007; Gomes, Matsuura \& Bhambhani, 2012).

421 An interesting study has recently investigated the acute effect of blood flow restriction (created

422 artificially using an inflatable cuff) during exercise, not only on muscle oxygenation, but also on

423 brain prefrontal cortex oxygenation (Ganesan et al., 2015).

424 Similarly to that study (Ganesan et al., 2015), our data suggests that the pronounced decrement in 425 muscle oxygenation can modulate exercise-induced changes in prefrontal cortex oxygenation, 426 which was proposed to be related to the perception of fatigue (RPE) (Pereira, Gomes \& 427 Bhambhani, 2009).

428 The results of the present study support the complementary interaction between the ipsilateral and 429 contralateral cortex during exercise to fatigue. In fact, we found that the increase of oxyhemoglobin 
430 in the contralateral lobe (and the consequent decrease of deoxyhemoglobin) was accompanied by

431 a smaller but consistent increase in the ipsilateral lobe. We also found that the change in

432 oxygenation was higher in the contralateral compared to the ipsilateral prefrontal lobe in each of

433 the three sets (Figure 5 and significant main effect of lobe shown in Table 1). The fact that

434 oxygenation increased in both prefrontal cortices, albeit the increase was lower for the ipsilateral

435 than the contralateral, is in line with the findings of a previous study (Kuboyama \& Shibuya, 2015),

436 which revealed an increase in oxyhemoglobin accompanied by a small decrease in

437 deoxyhemoglobin in both hemispheres during prolonged fatiguing repetitive handgrip exercise

438 performed at maximal voluntary contraction (Kuboyama \& Shibuya, 2015). However, despite

439 changes in both hemispheres, there was higher delayed oxygenation in the ipsilateral compared to

440 the contralateral cortex, thus suggesting that unilateral resistance exercise has a stronger

441 association with the contralateral rather than the ipsilateral lobe (Kuboyama \& Shibuya, 2015).

442 Our data provided evidence of a complementary interaction between the ipsilateral and 443 contralateral cortex during fatiguing exercise (Benwell, Mastaglia \& Thickbroom, 2006; Shibuya

444 \& Kuboyama, 2007), as well as a higher involvement of the contralateral prefrontal cortex 445 compared to the ipsilateral.

447 Limitations and further directions of research

448 It is possible that the variability of some oxygenation parameters in muscle, but especially in the 449 prefrontal cortex, among subjects may have hampered our ability to detect differences between 450 conditions in the second and third set of exercise. Although our sample size was found to be 451 sufficient, further studies will have to consider such variability by increasing the sample size to 452 achieve more statistical power. Furthermore, despite a previous study showing that there were no 
453 differences between males and females in muscle and cerebral oxygenation during resistance

454 exercise (Gomes, Matsuura \& Bhambhani, 2012), we put in evidence that our findings cannot be 455 surely extended also to male subjects. Thus, future studies will be addressed to compare exercise456 associated oxygenation changes of muscle and prefrontal cortex in male and female subjects, as 457 well as in subjects with different training levels.

458 However, it is important to notice that this study has been conducted using a frequency-domain 459 NIRS instrument that allowed us to carry out accurate measurements of absolute concentrations of 460 oxyhemoglobin and deoxyhemoglobin, thus also quantifying total hemoglobin and saturation. This 461 was useful in order to obtain a detailed portrait of hemodynamic changes in both muscle and the 462 prefrontal cortex related to resistance exercise with different speeds of movement. In addition, a 463 comparison between oxygenation changes in the contralateral and ipsilateral prefrontal cortex was 464 performed.

465 Finally, we suggest that further investigations should consider a combination of different 466 techniques to further explore the underlying physiological mechanisms of resistance exercise with 467 different speeds of movement, such as muscle activity by electromyography and tissue perfusion 468 by infrared thermography (Formenti et al., 2016). We also recommend the use of a full-head fNIRS 469 helmet to elucidate the response of different brain regions to resistance exercise performed to 470 fatigue.

472 Conclusions

473 In summary, the findings of the present study indicate that exercising with slow speed of movement 474 induces higher muscle deoxygenation than exercising with normal speed of movement. 475 Specifically, the exercise with a repetition duration lasting $10 \mathrm{~s}(5 \mathrm{~s}$ eccentric and 5 s concentric 
476 phase) was associated with larger muscle deoxygenation compared to the exercise with faster

477 movement ( $3 \mathrm{~s}$ and $1 \mathrm{~s}$ for each phase). Since the lack of oxygen in muscle can maximize the levels

478 of metabolic stress, which has a primary role in stimulating hypertrophy, manipulating the speed

479 of movement, and the related repetition duration, may be useful when planning resistance-training

480 programs to increase the metabolic stress associated to exercise.

481 Furthermore, we found a consistent oxygenation increase in both the right and left prefrontal lobes, 482 and that oxygenation changes were higher when exercising with slow movement. In addition, 483 higher changes in oxygenation were found in the contralateral rather than the ipsilateral prefrontal 484 cortex. These results provide further evidence of the complementary interactions between the 485 ipsilateral and contralateral prefrontal cortex during fatiguing resistance exercise, and that the 486 speed of movement has an impact on prefrontal cortex oxygenation.

488 Acknowledgements

489 Special thanks to Ben Oliver for proof-reading the manuscript and Antonello Chiarelli PhD for his 490 technical support.

491

\section{References}

493 Bandrivskyy A., Bernjak A., McClintock P., Stefanovska A. 2004. Wavelet phase coherence 494 analysis: application to skin temperature and blood flow. Cardiovascular Engineering: An International Journal 4:89-93. DOI: 10.1023/B:CARE.0000025126.63253.43.

van Beekvelt MC., Borghuis MS., van Engelen BG., Wevers RA., Colier WN. 2001. Adipose tissue thickness affects in vivo quantitative near-IR spectroscopy in human skeletal muscle. Clinical Science 101:21-28. 
499 Benwell NM., Mastaglia FL., Thickbroom GW. 2006. Reduced functional activation after 500 fatiguing exercise is not confined to primary motor areas. Experimental Brain Research 175:575-583. DOI: 10.1007/s00221-006-0573-9.

502 Chiarelli AM., Maclin EL., Fabiani M., Gratton G. 2015a. A kurtosis-based wavelet algorithm for 503 motion artifact correction of fNIRS data. NeuroImage 112:128-137. DOI: 10.1016/j.neuroimage.2015.02.057.

505

506

507

508

509

510

511

512

513

514

515

516

517

518

519

520

Chiarelli AM., Maclin EL., Low KA., Fabiani M., Gratton G. 2015b. Comparison of procedures for co-registering scalp-recording locations to anatomical magnetic resonance images. Journal of Biomedical Optics 20:016009. DOI: 10.1117/1.JBO.20.1.016009.

Chiarelli AM., Maclin EL., Low KA., Mathewson KE., Fabiani M., Gratton G. 2016. Combining energy and Laplacian regularization to accurately retrieve the depth of brain activity of diffuse optical tomographic data. Journal of Biomedical Optics 21:036008-036008. DOI: 10.1117/1.JBO.21.3.036008.

Cohen J. 1988. Statistical power analysis for the behavioral sciences. Academic Press.

Colier WN., Quaresima V., Oeseburg B., Ferrari M. 1999. Human motor-cortex oxygenation changes induced by cyclic coupled movements of hand and foot. Experimental Brain Research 129:457-461.

Day ML., McGuigan MR., Brice G., Foster C. 2004. Monitoring exercise intensity during resistance training using the session RPE scale. Journal of Strength and Conditioning Research 18:353-358. DOI: 10.1519/R-13113.1.

Felici F., Quaresima V., Fattorini L., Sbriccoli P., Filligoi GC., Ferrari M. 2009. Biceps brachii myoelectric and oxygenation changes during static and sinusoidal isometric exercises. 
521

522

523 Fernandes IA., Mattos JD., Campos MO., Machado AC., Rocha MP., Rocha NG., Vianna LC., 524

525

526

527

528

529

530

531

532

533

534

535

536

537

538

539

540

541

542

Journal of Electromyography and Kinesiology 19:e1-e11. DOI: 10.1016/j.jelekin.2007.07.010. Nobrega ACL. 2016. Selective $\alpha_{1}$-adrenergic blockade disturbs the regional distribution of cerebral blood flow during static handgrip exercise. American Journal of PhysiologyHeart and Circulatory Physiology 310:H1541-H1548. DOI: 10.1152/ajpheart.00125.2016.

Ferrante S., Contini D., Spinelli L., Pedrocchi A., Torricelli A., Molteni F., Ferrigno G., Cubeddu R. 2009. Monitoring muscle metabolic indexes by time-domain near-infrared spectroscopy during knee flex-extension induced by functional electrical stimulation. Journal of Biomedical Optics 14:044011. DOI: 10.1117/1.3183802.

Formenti D., Ludwig N., Trecroci A., Gargano M., Michielon G., Caumo A., Alberti G. 2016. Dynamics of thermographic skin temperature response during squat exercise at two different speeds. Journal of Thermal Biology 59:58-63. DOI: 10.1016/j.jtherbio.2016.04.013.

Gandevia SC. 2001. Spinal and supraspinal factors in human muscle fatigue. Physiological Reviews 81:1725-1789. DOI: 10.1152/physrev.2001.81.4.1725.

Ganesan G., Cotter JA., Reuland W., Cerussi AE., Tromberg BJ., Galassetti P. 2015. Effect of blood flow restriction on tissue oxygenation during knee extension. Medicine \& Science in Sports \& Exercise 47:185-193. DOI: 10.1249/MSS.0000000000000393.

Gomes PSC., Matsuura C., Bhambhani YN. 2012. Effects of hypoxia on cerebral and muscle haemodynamics during knee extensions in healthy subjects. European Journal of Applied Physiology 113:13-23. DOI: 10.1007/s00421-012-2408-7. 
544 Headley SA., Henry K., Nindl BC., Thompson BA., Kraemer WJ., Jones MT. 2011. Effects of

545 lifting tempo on one repetition maximum and hormonal responses to a bench press

546 protocol. Journal of Strength and Conditioning Research 25:406-413. DOI: 10.1519/JSC.0b013e3181bf053b.

548

549

550

551

552

553

554

555

556

557

558

559

560

561

562

563

564

565

566

Homan RW., Herman J., Purdy P. 1987. Cerebral location of international 10-20 system electrode placement. Electroencephalography and Clinical Neurophysiology 66:376-382. DOI: 10.1016/0013-4694(87)90206-9.

Hueber DM., Fantini S., Cerussi AE., Barbieri BB. 1999. New optical probe designs for absolute (self-calibrating) NIR tissue hemoglobin measurements. In: Optical Tomography and Spectroscopy of Tissue III. International Society for Optics and Photonics, 618-632. DOI: 10.1117/12.356784.

Husmann F., Mittlmeier T., Bruhn S., Zschorlich V., Behrens M. 2017. Impact of blood flow restriction exercise on muscle fatigue development and recovery. Medicine \& Science in Sports \& Exercise. DOI: 10.1249/MSS.0000000000001475.

Kawada S., Ishii N. 2005. Skeletal muscle hypertrophy after chronic restriction of venous blood flow in rats. Medicine \& Science in Sports \& Exercise 37:1144-1150.

Kohno S., Miyai I., Seiyama A., Oda I., Ishikawa A., Tsuneishi S., Amita T., Shimizu K. 2007. Removal of the skin blood flow artifact in functional near-infrared spectroscopic imaging data through independent component analysis. Journal of Biomedical Optics 12:062111. DOI: $10.1117 / 1.2814249$.

Kraemer WJ., Ratamess NA. 2004. Fundamentals of resistance training: progression and exercise prescription. Medicine \& Science in Sports \& Exercise 36:674-688. DOI: 10.1249/01.MSS.0000121945.36635.61. 
567 Kuboyama N., Shibuya K. 2015. Ipsi- and contralateral frontal cortex oxygenation during handgrip 568 task does not follow decrease on maximal force output. Journal of Physiological 569 Anthropology 34:37. DOI: 10.1186/s40101-015-0077-z.

570 Kvandal P., Landsverk SA., Bernjak A., Stefanovska A., Kvernmo HD., Kirkebøen KA. 2006.

571 Low-frequency oscillations of the laser Doppler perfusion signal in human skin. 572 Microvascular Research 72:120-127. DOI: 10.1016/j.mvr.2006.05.006.

573 Loenneke JP., Fahs CA., Rossow LM., Abe T., Bemben MG. 2012. The anabolic benefits of 574

575 venous blood flow restriction training may be induced by muscle cell swelling. Medical hypotheses 78:151-154. DOI: 10.1016/j.mehy.2011.10.014.

Matsuura C., Gomes PSC., Haykowsky M., Bhambhani Y. 2011. Cerebral and muscle oxygenation changes during static and dynamic knee extensions to voluntary fatigue in healthy men and women: a near infrared spectroscopy study. Clinical Physiology and Functional Imaging 31:114-123. DOI: 10.1111/j.1475-097X.2010.00986.x.

Moritani T., Sherman WM., Shibata M., Matsumoto T., Shinohara M. 1992. Oxygen availability and motor unit activity in humans. European Journal of Applied Physiology and Occupational Physiology 64:552-556.

Pearson SJ., Hussain SR. 2015. A review on the mechanisms of blood-flow restriction resistance training-induced muscle hypertrophy. Sports Medicine 45:187-200. DOI: 10.1007/s40279-014-0264-9.

Pereira MIR., Gomes PSC., Bhambhani YN. 2009. Acute effects of sustained isometric knee 588 extension on cerebral and muscle oxygenation responses. Clinical Physiology and 588 Functional Imaging 29:300-308. DOI: 10.1111/j.1475-097X.2009.00870.x. 
589 Richardson JTE. 2011. Eta squared and partial eta squared as measures of effect size in educational $590 \quad$ research. Educational Research Review 6:135-147. DOI: 10.1016/j.edurev.2010.12.001.

591 Schoenfeld BJ., Ogborn DI., Krieger JW. 2015. Effect of repetition duration during resistance 592 593 training on muscle hypertrophy: a systematic review and meta-analysis. Sports Medicine 45:577-585. DOI: 10.1007/s40279-015-0304-0.

594 595 596 597 598 599 600 601 602 603 604 605 606 607 Tamaki T., Uchiyama S., Tamura T., Nakano S. 1994. Changes in muscle oxygenation during 608 609 weight-lifting exercise. European Journal of Applied Physiology and Occupational Physiology 68:465-469. DOI: 10.1007/BF00599514. 
610 Tanimoto M., Ishii N. 2006. Effects of low-intensity resistance exercise with slow movement and 611 tonic force generation on muscular function in young men. Journal of Applied Physiology 612 100:1150-1157. DOI: 10.1152/japplphysiol.00741.2005.

613 Tanimoto M., Madarame H., Ishii N. 2005. Muscle oxygenation and plasma growth hormone 614 concentration during and after resistance exercise: Comparison between" KAATSU" and 615 other types of regimen. International Journal of KAATSU Training Research 1:51-56.

616 Tanimoto M., Sanada K., Yamamoto K., Kawano H., Gando Y., Tabata I., Ishii N., Miyachi M. 617 2008. Effects of whole-body low-intensity resistance training with slow movement and 618 tonic force generation on muscular size and strength in young men. Journal of Strength 619 and Conditioning Research 22:1926-1938.

620 Watanabe Y., Tanimoto M., Ohgane A., Sanada K., Miyachi M., Ishii N. 2013. Increased muscle 621 size and strength from slow-movement, low-intensity resistance exercise and tonic force 622 generation. Journal of Aging and Physical Activity 21:71-84.

623 Yeung SS., Ting KH., Hon M., Fung NY., Choi MM., Cheng JC., Yeung EW. 2016. Effects of 624 cold water immersion on muscle oxygenation during repeated bouts of fatiguing exercise: 625 a randomized controlled study. Medicine 95.

626 


\section{Table $\mathbf{1}$ (on next page)}

F-values, partial eta squared $\left(\eta_{p}{ }^{2}\right)$ and observed power of Two-way ANOVA repeated measures (Lobe $x$ Condition) for each set.

$\Delta \mathrm{Hb}$ (deoxyhemoglobin change), $\Delta \mathrm{HbO}$ (oxyhemoglobin change), $\Delta \mathrm{HbT}$ (total hemoglobin change), and $\Delta \mathrm{StO}_{2}$ (oxygen saturation change). ${ }^{*} \mathrm{p}<0.05 ; * * p<0.01$. 


\begin{tabular}{|c|c|c|c|c|c|c|c|c|c|}
\hline \multirow[b]{2}{*}{ Set1 } & \multicolumn{3}{|l|}{ Lobe } & \multicolumn{3}{|c|}{ Condition } & \multicolumn{3}{|c|}{ Lobe $\mathrm{x}$ Condition } \\
\hline & $\mathrm{F}_{(1,7)}$ & $\eta_{\mathrm{P}}^{2}$ & $\begin{array}{l}\text { Observed Power } \\
(1-\beta \text { err prob) }\end{array}$ & $\mathrm{F}_{(2,14)}$ & $\eta_{\mathrm{P}}^{2}$ & $\begin{array}{l}\text { Observed Power } \\
\text { (1- } \beta \text { err prob) }\end{array}$ & $\mathrm{F}_{(2,14)}$ & $\eta_{\mathrm{P}}^{2}$ & $\begin{array}{l}\text { Observed Power } \\
\text { (1- } \beta \text { err prob) }\end{array}$ \\
\hline$\Delta \mathrm{Hb}$ & $6.907^{*}$ & 0.21 & 0.51 & $5.955^{*}$ & 0.29 & 0.83 & $4.174^{*}$ & 0.19 & 0.59 \\
\hline$\Delta \mathrm{HbO}$ & $7.821 *$ & 0.24 & 0.72 & $5.263 *$ & 0.28 & 0.81 & 2.808 & 0.11 & 0.34 \\
\hline$\Delta \mathrm{HbT}$ & $6.802 *$ & 0.21 & 0.51 & $5.426^{*}$ & 0.28 & 0.81 & 3.567 & 0.17 & 0.52 \\
\hline$\Delta \mathrm{StO}_{2}$ & $6.913^{*}$ & 0.20 & 0.61 & $9.977 * *$ & 0.42 & 0.98 & 2.117 & 0.09 & 0.28 \\
\hline
\end{tabular}

\begin{tabular}{|c|c|c|c|c|c|c|c|c|c|}
\hline \multirow[b]{2}{*}{ Set2 } & \multicolumn{3}{|l|}{ Lobe } & \multicolumn{3}{|c|}{ Condition } & \multicolumn{3}{|c|}{ Lobe $\mathrm{x}$ Condition } \\
\hline & $\mathrm{F}_{(1,7)}$ & $\eta_{\mathrm{P}}^{2}$ & $\begin{array}{l}\text { Observed Power } \\
(1-\beta \text { err prob) }\end{array}$ & $\mathrm{F}_{(2,14)}$ & $\eta_{\mathrm{P}}^{2}$ & $\begin{array}{l}\text { Observed Power } \\
\text { (1- } \beta \text { err prob) }\end{array}$ & $\mathrm{F}_{(2,14)}$ & $\eta_{\mathrm{P}}^{2}$ & $\begin{array}{l}\text { Observed Power } \\
\text { (1- } \beta \text { err prob) }\end{array}$ \\
\hline$\Delta \mathrm{Hb}$ & $5.53 *$ & 0.19 & 0.59 & 0.853 & 0.02 & 0.09 & 1.811 & 0.09 & 0.28 \\
\hline$\Delta \mathrm{HbO}$ & $8.534 *$ & 0.22 & 0.67 & 2.285 & 0.08 & 0.24 & 0.649 & 0.02 & 0.09 \\
\hline$\Delta \mathrm{HbT}$ & $19.2 * *$ & 0.39 & 0.95 & 1.359 & 0.06 & 0.19 & 1.81 & 0.09 & 0.28 \\
\hline$\Delta \mathrm{StO}_{2}$ & $9.17 *$ & 0.28 & 0.81 & 1.59 & 0.06 & 0.19 & 0.139 & 0.001 & 0.05 \\
\hline
\end{tabular}

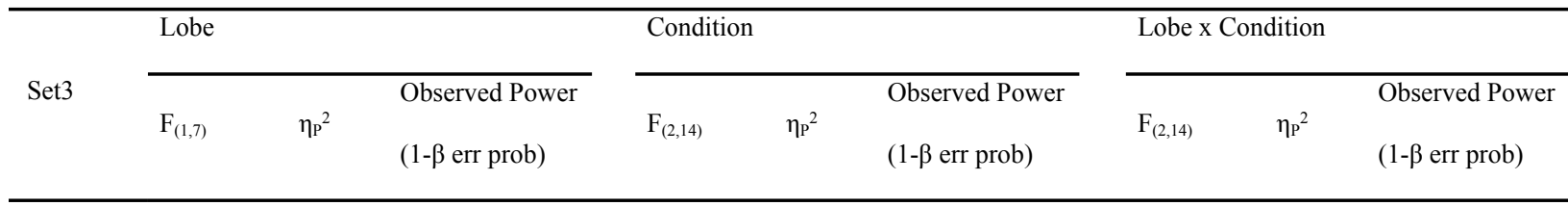

\begin{tabular}{|c|c|c|c|c|c|c|c|c|c|}
\hline$\overline{\Delta \mathrm{Hb}}$ & $10.55^{*}$ & 0.29 & 0.83 & 1.638 & 0.06 & 0.19 & 0.706 & 0.02 & 0.09 \\
\hline$\Delta \mathrm{HbO}$ & $7.449 *$ & 0.24 & 0.72 & 1.158 & 0.04 & 0.14 & 0.004 & 0.001 & 0.05 \\
\hline$\Delta \mathrm{HbT}$ & 2.493 & 0.09 & 0.28 & 3.71 & 0.17 & 0.53 & 0.355 & 0.006 & 0.06 \\
\hline$\Delta \mathrm{StO}_{2}$ & $5.095 *$ & 0.19 & 0.59 & 3.6 & 0.17 & 0.53 & 0.323 & 0.006 & 0.06 \\
\hline
\end{tabular}

$1 \longdiv { \text { Table 1. F-values, partial eta squared } ( \eta _ { \mathrm { P } } { } ^ { 2 } ) \text { and observed power of Two-way ANOVA repeated } }$

2 measures (Lobe $\mathrm{x}$ Condition) for each set. $\Delta \mathrm{Hb}$ (deoxyhemoglobin change), $\Delta \mathrm{HbO}$

3 (oxyhemoglobin change), $\Delta \mathrm{HbT}$ (total hemoglobin change), and $\Delta \mathrm{StO}_{2}$ (oxygen saturation

4 change). ${ }^{*} \mathrm{p}<0.05 ; * *_{\mathrm{p}}<0.01$. 
Figure 1

Schematic representation of the experimental design. The preliminary session and each testing session were performed in separate days.

1s exercise: 1s concentric and 1s eccentric phase. 3s exercise: $3 \mathrm{~s}$ concentric and $3 \mathrm{~s}$ eccentric phase. $5 \mathrm{~s}$ exercise: $5 \mathrm{~s}$ concentric and $5 \mathrm{~s}$ eccentric phase.

\section{PRELIMINARY SESSION}

Anthropometric measurement

1 maximal repetition (1RM) test

Familiarization with $1 \mathrm{~s}, 3 \mathrm{~s}$, and $5 \mathrm{~s}$ pace

TYPICAL TESTING SESSION

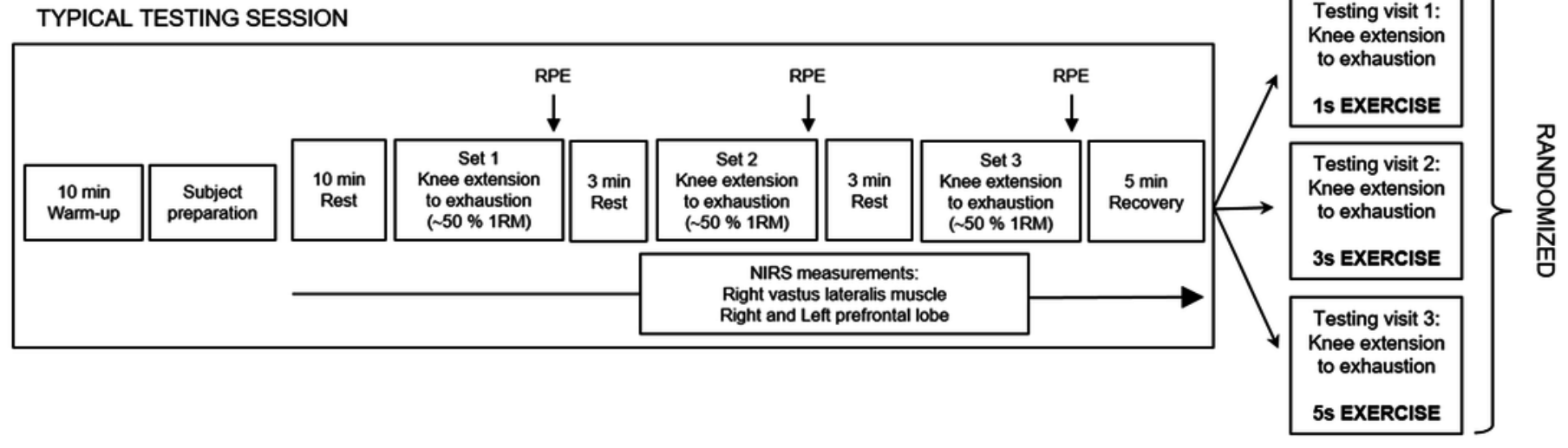




\section{Figure 2}

Effect of different speeds of movement on maximal number of repetitions (A) and on RPE (B) during three sets of knee extension exercise.

Values are mean \pm SD. Significant pairwise comparisons between conditions are shown. $\# p<0.05$ for interaction set $x$ condition. ${ }^{*} p<0.05, * * p<0.01, * * * p<0.001$ for pairwise comparisons between conditions. 1s: 1s concentric and 1s eccentric phase. 3s: 3 s concentric and 3s eccentric phase. 5s: 5s concentric and 5s eccentric phase.

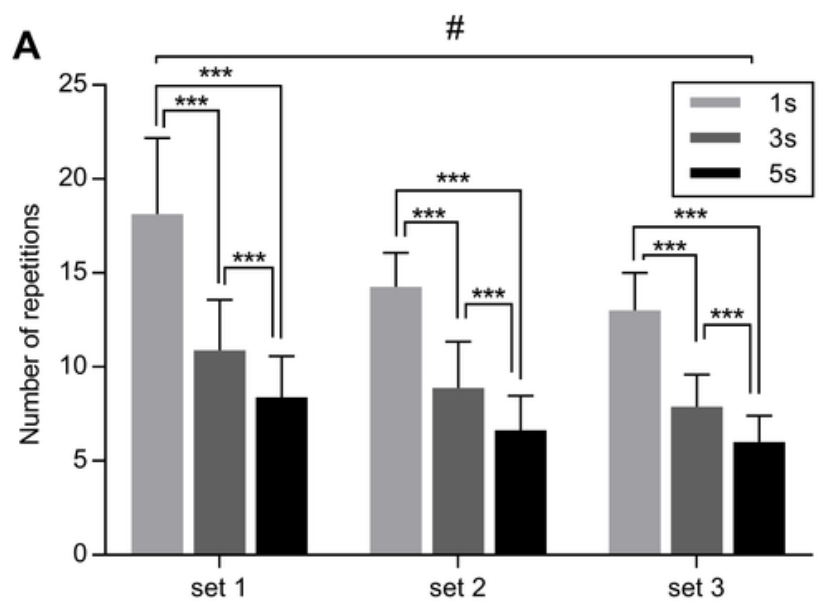

B

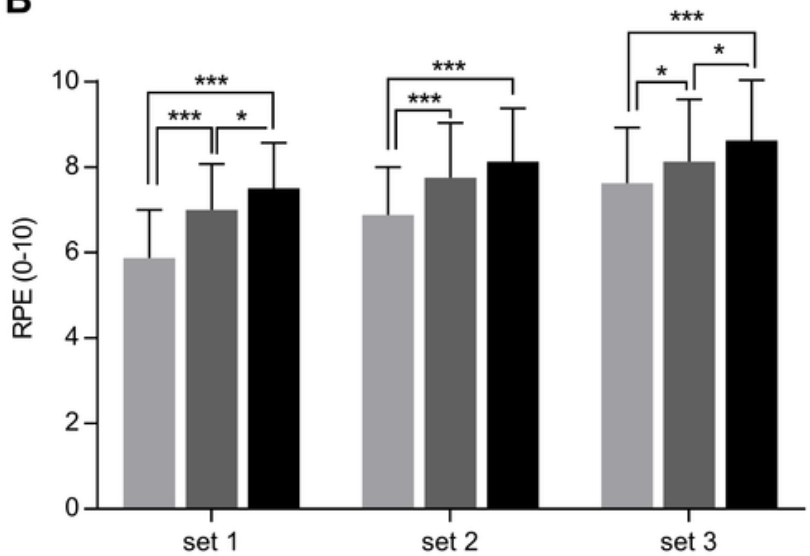


Figure 3

Example of muscle and cerebral oxygenation during exercise.

A. Muscle hemodynamic responses for $\mathrm{Hb}$ (deoxyhemoglobin) and $\mathrm{HbO}$ (oxyhemoglobin) during the set2 of each condition (1s, $3 \mathrm{~s}$ and 5 s exercise) of knee extension exercise in a representative subject. B. Prefrontal cortex hemodynamic responses for $\mathrm{Hb}$ and $\mathrm{HbO}$ during the set2 of the $3 \mathrm{~s}$ exercise of knee extension in a representative subject.

A. $\mathrm{Hb} \& \mathrm{HbO}$ in muscle

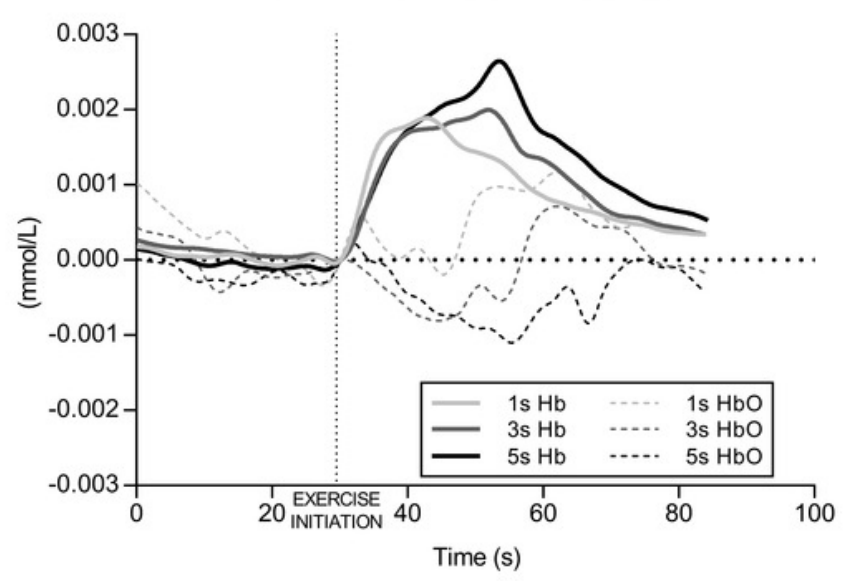

B. $\mathrm{Hb} \& \mathrm{HbO}$ in prefrontal cortex

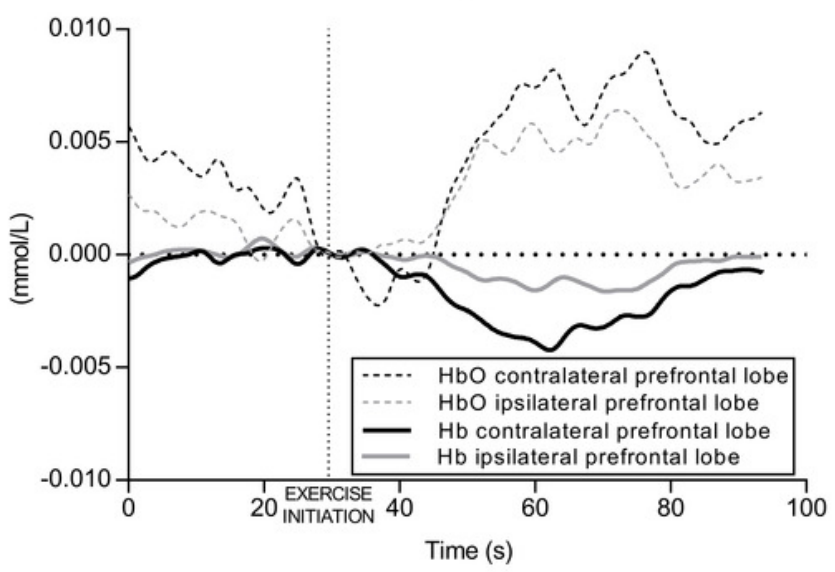




\section{Figure 4}

Effect of different speeds of movement on muscle oxygenation change during three sets of knee extension exercise.

$\Delta \mathrm{Hb}$ : deoxyhemoglobin change $(\mathrm{A}), \Delta \mathrm{HbO}$ : oxyhemoglobin change $(\mathrm{B}), \Delta \mathrm{HbT}$ : total hemoglobin change (C), $\Delta \mathrm{StO}_{2}$ : oxygen saturation change (D). The box shows the median and interquartile range, with the whiskers indicating the range of values. Dots are values of each subject. When significant main effect of condition was found, significant pairwise comparisons between conditions are shown. $\# p<0.05$ for interaction set $x$ condition. ${ }^{*} p<0.05,{ }^{*} p<0.01$ for pairwise comparisons between conditions. 1s: 1s concentric and 1s eccentric phase. 3s: $3 s$ concentric and 3s eccentric phase. 5s: $5 s$ concentric and $5 s$ eccentric phase.

\section{Muscle oxygenation}

A

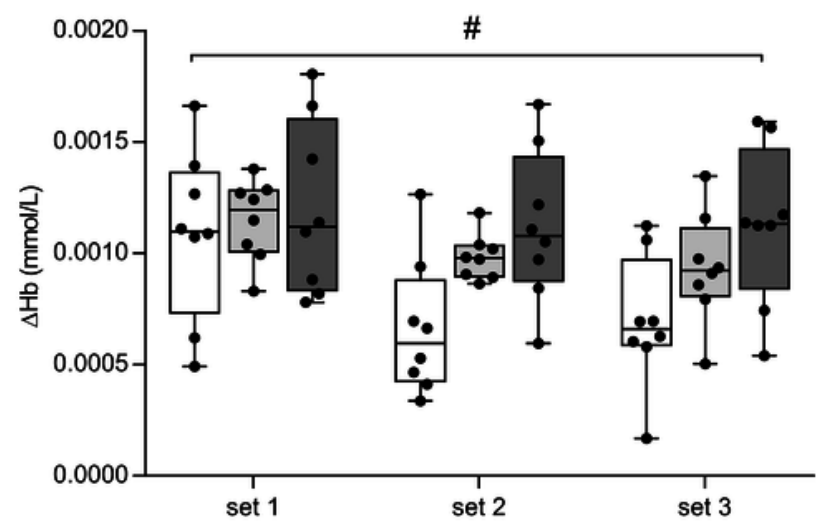

C

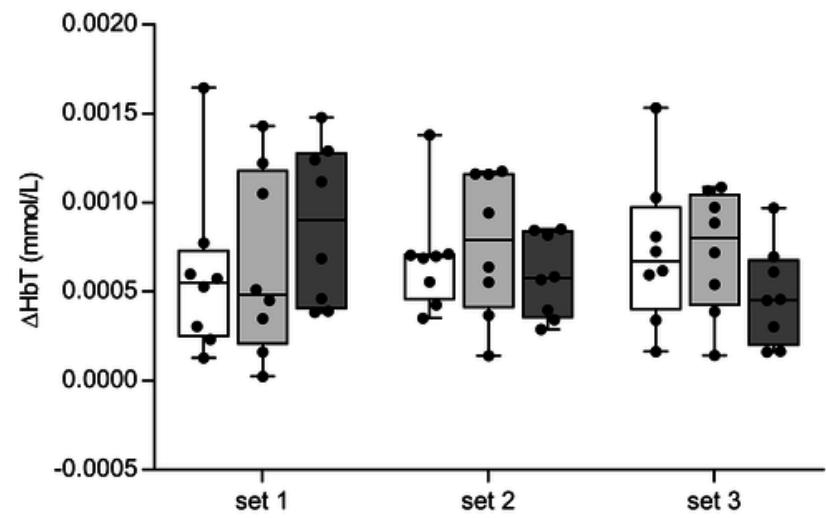

B

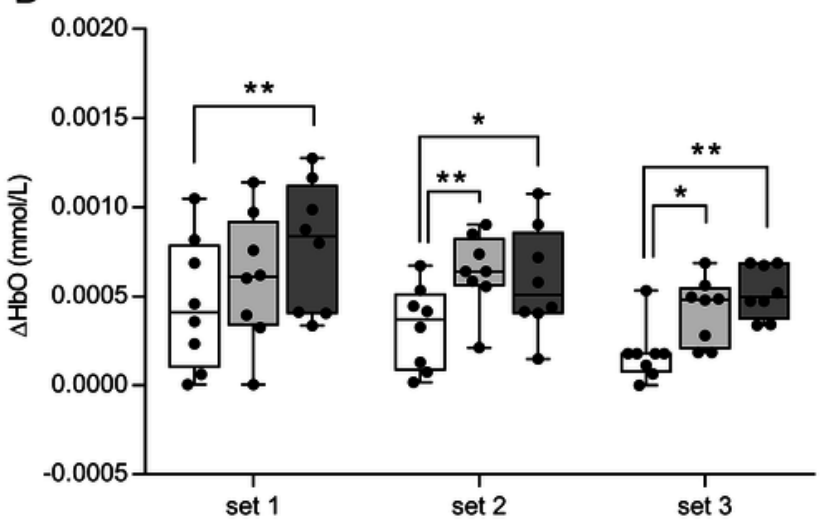

D

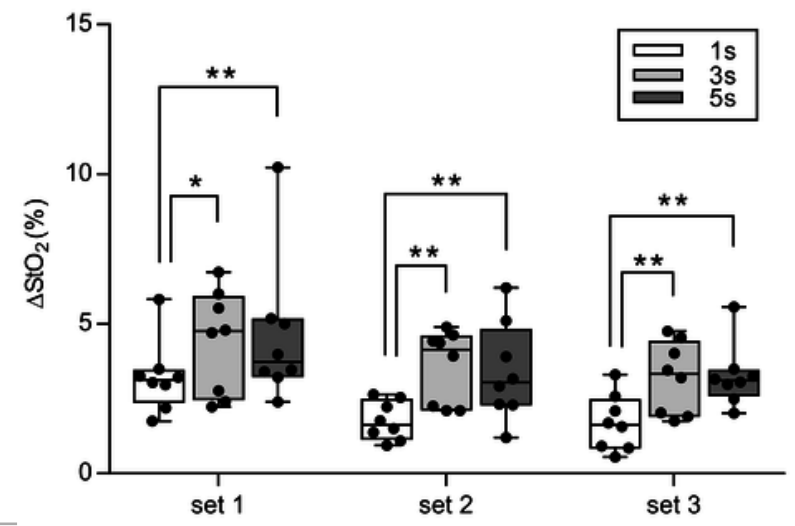




\section{Figure 5}

Effect of different speeds of movement on cerebral oxygenation change in left and right prefrontal lobe during three sets of knee extension exercise.

$\Delta \mathrm{Hb}$ : deoxyhemoglobin change (A), $\Delta \mathrm{HbO}$ : oxyhemoglobin change (B), $\Delta \mathrm{HbT}$ : total hemoglobin change (C), $\Delta \mathrm{StO}_{2}$ : oxygen saturation change (D). The box shows the median and interquartile range, with the whiskers indicating the range of values. Dots are values of each subject. Significant pairwise comparisons between lobes and between conditions are shown. $\# p<0.05$ for interaction lobe $x$ condition. $* p<0.05, * * p<0.01, * * * p<0.001$. 1s: 1s concentric and $1 \mathrm{~s}$ eccentric phase. $3 \mathrm{~s}$ : $3 \mathrm{~s}$ concentric and $3 \mathrm{~s}$ eccentric phase. $5 \mathrm{~s}$ : $5 \mathrm{~s}$ concentric and $5 \mathrm{~s}$ eccentric phase. 

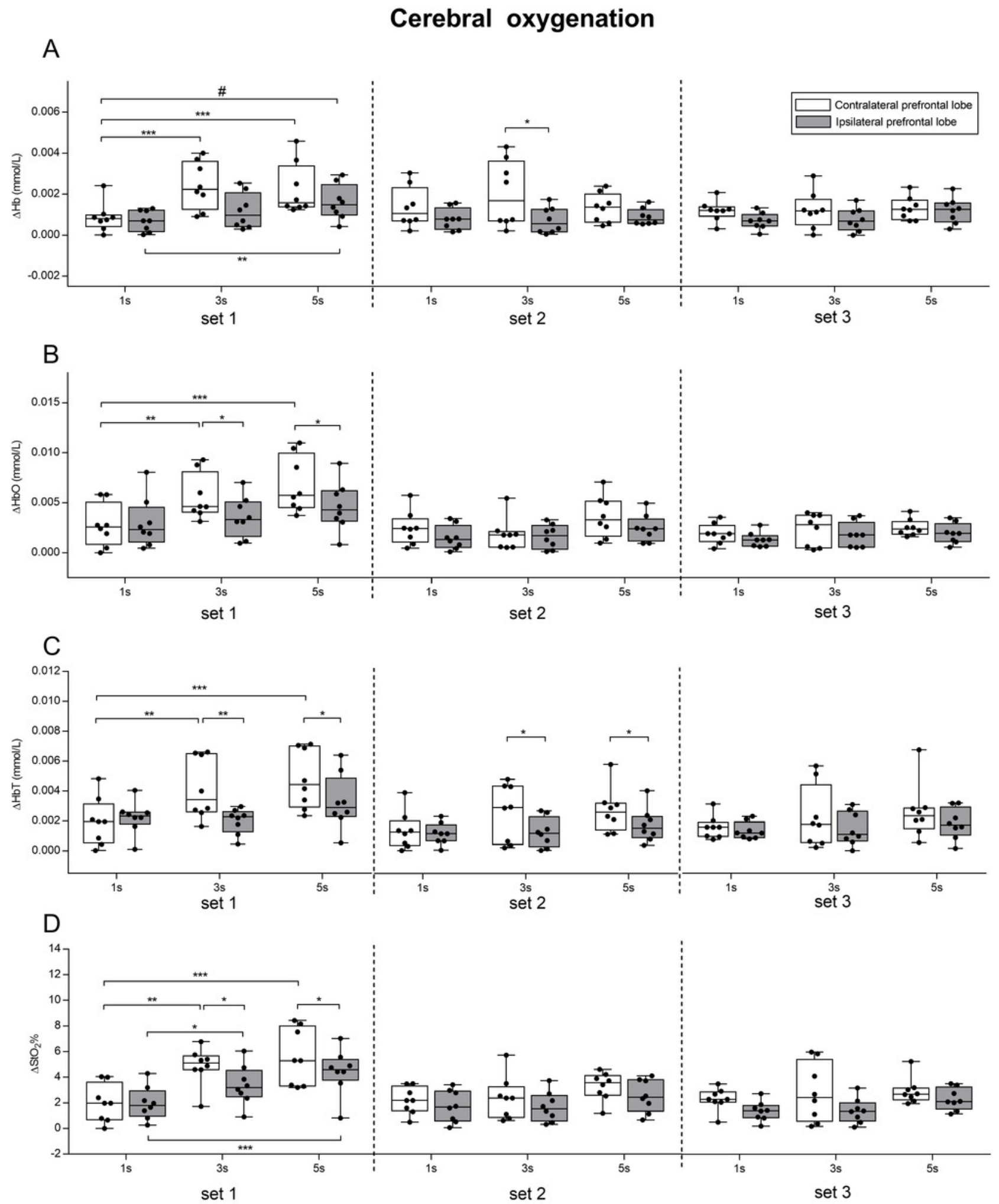